\title{
openheart The systolic paradox in hypertrophic cardiomyopathy
}

Trine F Haland, ${ }^{1,2}$ Nina E Hasselberg, ${ }^{1,2}$ Vibeke Marie Almaas, ${ }^{1,2}$ Lars A Dejgaard, ${ }^{1,2}$ Jørg Saberniak, ${ }^{1,2}$ Ida S Leren, ${ }^{1,2}$ Knut Erik Berge ${ }^{3}$ Kristina H Haugaa, ${ }^{1,2}$ Thor Edvardsen ${ }^{1,2}$

To cite: Haland TF, Hasselberg NE, Almaas VM, et al. The systolic paradox in hypertrophic cardiomyopathy. Open Heart 2017;4:e000571. doi:10.1136/ openhrt-2016-000571

Received 14 November 2016 Revised 8 February 2017 Accepted 21 March 2017
CrossMark

${ }^{1}$ Department of Cardiology, Institute for Surgical Research and Center for Cardiological Innovation, Oslo University Hospital, Rikshospitalet, Oslo, Norway

${ }^{2}$ University of Oslo, Norway ${ }^{3}$ Department of Medical Genetics, Unit for Cardiac and Cardiovascular Genetics, 0slo University Hospital, Ullevål, Oslo, Norway

Correspondence to Dr Thor Edvardsen; thor edvardsen@medisin.uio.no

\section{ABSTRACT}

Objective We explored cardiac volumes and the effects on systolic function in hypertrophic cardiomyopathy (HCM) patients with left ventricular hypertrophy $(\mathrm{HCM} \mathrm{LVH}+)$ and genotype-positive patients without left ventricular hypertrophy (HCM LVH-).

Methods We included 180 HCM LVH+, 100 HCM LVHpatients and 80 healthy individuals. End-Diastolic Volume Index (EDVI), End-Systolic Volume Index (ESVI) and ejection fraction (EF) were assessed by echocardiography. Left ventricular (LV) global longitudinal strain (GLS) was measured by speckle tracking echocardiography. Results EDVI and ESVI were significantly smaller in HCM $\mathrm{LVH}+$ compared with HCM LVH- patients $(41 \pm 14 \mathrm{~mL} /$ $\mathrm{m}^{2}$ vs $49 \pm 13 \mathrm{~mL} / \mathrm{m}^{2}$ and $16 \pm 7 \mathrm{~mL} / \mathrm{m}^{2}$ vs $19 \pm 6 \mathrm{~mL} / \mathrm{m}^{2}$, respectively, both $p<0.001$ ) and in healthy individuals $\left(41 \pm 14 \mathrm{~mL} / \mathrm{m}^{2}\right.$ vs $57 \pm 14 \mathrm{~mL} / \mathrm{m}^{2}$ and $16 \pm 7 \mathrm{~mL} / \mathrm{m}^{2}$ vs $23 \pm 9 \mathrm{~mL} / \mathrm{m}^{2}$, respectively, both $\left.\mathrm{p}<0.001\right)$. HCM LVHpatients had significantly lower EDVI and ESVI compared with healthy individuals $\left(49 \pm 13 \mathrm{~mL} / \mathrm{m}^{2}\right.$ vs $57 \pm 14 \mathrm{~mL}$ $\mathrm{m}^{2}$ and $19 \pm 6 \mathrm{~mL} / \mathrm{m}^{2}$ vs $23 \pm 9 \mathrm{~mL} / \mathrm{m}^{2}$, both $\left.\mathrm{p}<0.001\right)$. EF was similar $(61 \% \pm 7 \%$ vs $60 \% \pm 8 \%$ vs $61 \% \pm 6 \%, p=0.43)$ in the HCM LVH+, HCM LVH- and healthy individuals, despite significantly worse GLS in the HCM LVH+ $(-16.4 \% \pm 3.7 \%$ vs $-21.3 \% \pm 2.4 \%$ vs $-22.3 \% \pm 3.7 \%$, $\mathrm{p}<0.001)$. GLS was worse in the HCM LVH- compared with healthy individuals in pairwise comparison $(p=0.001)$. Decrease in ESVI was closely related to EF in $\mathrm{HCM} \mathrm{LVH+}$ and HCM LVH- $(R=0.45, p<0.001$ and $R=0.43, p<0.001)$ as expected, but there was no relationship with GLS $(\mathrm{R}=0.02, \mathrm{p}=0.77$ and $\mathrm{R}=0.11, \mathrm{p}=0.31)$. Increased maximal wall thickness (MWT) correlated significantly with worse GLS ( $R=0.58, p<0.001)$, but not with $E F(R=0.018$, $\mathrm{p}=0.30$ ) in the $\mathrm{HCM} \mathrm{LVH}+$ patients.

Conclusion HCM LVH+ had smaller cardiac volumes that could explain the preserved EF, despite worse GLS that was closely related to MWT. HCM LVH- had reduced cardiac volumes and subtle changes in GLS compared with healthy individuals, indicating a continuum of both volumetric and systolic changes present before increased MWT.

\section{INTRODUCTION}

Hypertrophic cardiomyopathy (HCM) is one of the most common inherited cardiomyopathies with an estimated prevalence of 1:500. HCM is characterised by the presence of increased left ventricular (LV) wall thickness that is not explained by abnormal loading conditions

\section{KEY MESSAGES}

What is already known about this subject? Hypertrophic cardiomyopathy patients with left ventricular hypertrophy (HCM LVH+) and genotypepositive patients without left ventricular hypertrophy (HCM LVH-) typically have normal or elevated ejection fraction (EF), despite decreased longitudinal function.

What does this study add?

Smaller cardiac volumes in patients with HCM are related to preserved EF in $\mathrm{HCM} \mathrm{LVH+} \mathrm{patients.}$ In HCMLVH - patients there were subtle changes in both volumetric and systolic changes, indicating a continuum of changes from the healthy individuals to HCM LVH- and HCM LVH+ patients. Global longitudinal strain (GLS) was closely related to maximal wall thickness in HCM LVH+ patients.

How might this impact on clinical practice? HCM LVH- patients with volumetric and systolic changes should be closely followed. GLS is a sensitive parameter for detecting systolic impairment and is related to increased development of the HCM disease.

with heterogeneous disease development and expression. Sarcomere mutations are identified in $60 \%-70 \%$ of HCM cases. ${ }^{1}$ Echocardiography is a valuable tool in diagnosis and follow-up of patients with HCM, evaluating morphology, structural abnormalities, haemodynamic disturbances and prognosis. ${ }^{2}{ }^{3} \mathrm{LV}$ systolic function is closely related to prognosis in cardiac diseases in general. ${ }^{4} \mathrm{LV}$ ejection fraction (EF) is based on volume measurements and is the most widely used metric of LV systolic function despite its inherent weaknesses. EF is typically normal in patients with HCM and may remain normal until the end stage of the disease. ${ }^{5} \mathrm{EF}$ is therefore considered inadequate to evaluate the indication for medical treatment and cardiac transplantation in $\mathrm{HCM}^{1}$. We know that patients with HCM have decreased longitudinal systolic function despite normal EF and that worse global longitudinal strain (GLS) is associated with poor 
cardiac outcomes. ${ }^{6-10}$ However, the mechanism behind normal EF in HCM patientswith reduced systolicfunction is not comprehensively described. A possible explanation is that the increased wall thickness results in lower volumes and alter the equation for EF ((end-diastolic volumeend-systolic volume)/end-diastolic volume).

Previous studies have also shown subtle changes in systolic function in genotype-positive patients without left \& ventricular hypertrophy(HCM LVH-) despite normal EF. ${ }^{11-13}$ However, we need more studies to identify morphological features in HCM LVH- patients to identify the patients who should be closely followed.

We aimed to study the relationship between volumetric changes and the paradox of normal EF and decreased longitudinal function by strain echocardiography in a large population of HCM patients with left ventricular hypertrophy (HCM LVH+) and HCM LVH-. We hypothesised that volumetric changes in HCM occur as a continuum from the HCM LVH- to the HCM LVH+.

\section{METHODS}

\section{Study population}

In this cross-sectional study, patients with HCM were consecutively included when appointed at the Unit of
Cardiac Genetic Disease in our department from 2005 to 2014.

It is known that $\mathrm{LV}$ mass can be normal in patients with $\mathrm{HCM},{ }^{14}$ and these patients were defined as phenotype positive if they had a ventricular maximal wall thickness (MWT) $\geq 15 \mathrm{~mm}$ with no other obvious explanation for the hypertrophy or MWT of $\geq 13 \mathrm{~mm}$ in the presence of a HCM-related mutation (HCM LVH+). ${ }^{1}$

Patients with HCM were defined as phenotype negative in the presence of a sarcomere mutation (HCM LVH-) with $\mathrm{LV}$ wall thickness $<13 \mathrm{~mm}$ and no history of cardiac symptoms.

All the patients underwent clinical examination and echocardiography.

Healthy individuals were invited to participate in the study from hospital staff, medical school and research laboratories, and age matched with HCM LVH+ patients. All participants gave written informed consent. The study complied with the Declaration of Helsinki and was approved by the Regional Committees for Medical Research Ethics.

\section{Genetic analyses}

DNA sequencing of the genes encoding the sarcomere proteins MYH7 (NM_000257.2), MYBPC3

Table 1 Clinical characteristics and echocardiographic findings in 80 healthy individuals, 100 HCM LVH- patients and 180 HCM LVH+ patients

\begin{tabular}{|c|c|c|c|c|}
\hline & $\begin{array}{l}\text { Healthy individuals } \\
(n=80)\end{array}$ & $\begin{array}{l}\text { HCM LVH- patients } \\
(n=100)\end{array}$ & $\begin{array}{l}\text { HCM LVH+ patients } \\
(n=180)\end{array}$ & p Value \\
\hline Age (years) & $54 \pm 15$ & $36 \pm 15^{*}$ & $53 \pm 17$ & $<0.001$ \\
\hline BMI $\left(\mathrm{kg} / \mathrm{m}^{2}\right)$ & $24 \pm 3$ & $24 \pm 4$ & $27 \pm 4^{*}+$ & $<0.001$ \\
\hline $\mathrm{BSA}\left(\mathrm{m}^{2}\right)$ & $1.9 \pm 0.2$ & $1.8 \pm 0.2$ & $1.9 \pm 0.2^{\dagger}$ & $<0.001$ \\
\hline Systolic BP (mm Hg) & $124 \pm 11$ & $127 \pm 18$ & $131 \pm 12$ & 0.27 \\
\hline Diastolic BP (mm Hg) & $71 \pm 11$ & $76 \pm 10$ & $79 \pm 13$ & 0.09 \\
\hline \multicolumn{5}{|l|}{ Echocardiography } \\
\hline MWT (mm) & $9 \pm 1$ & $8 \pm 1$ & $19 \pm 4^{* \dagger}$ & $<0.001$ \\
\hline IVSd (mm) & $8 \pm 2$ & $8 \pm 2$ & $16 \pm 4^{*} \dagger$ & $<0.001$ \\
\hline LVPWd & $8 \pm 2$ & $8 \pm 3$ & $10 \pm 3^{* \dagger}$ & $<0.001$ \\
\hline LV mass/BSA $\left(\mathrm{g} / \mathrm{m}^{2}\right)$ & $73 \pm 22$ & $71 \pm 15$ & $132 \pm 45^{* \dagger}$ & $<0.001$ \\
\hline
\end{tabular}

Values are represented as mean $\pm \mathrm{SD}$.

$p$ values are based on ANOVA and $\chi^{2}$ test.

${ }^{*} \mathrm{p}<0.05$ compared with healthy individuals.

$\dagger \mathrm{p}<0.05$ compared with HCM LVH- patients by Bonferroni post hoc correction.

ANOVA, analysis of variance; BMI, body mass index; BP, blood pressure; BPM, beats per minute; BSA, body surface area; HCM,

hypertrophic cardiomyopathy; HCM LVH-, HCM patients without increased wall thickness; HCM LVH+, HCM patients with increased wall thickness; MWT, maximal wall thickness; NYHA class, New York Heart Association functional classification; LV, Left ventricular; LVEDD, left ventricular end-diastolic diameter; LVESD, left ventricular end-systolic diameter; LVH, left ventricular hypertrophy; LVOT, left ventricle outflow tract; LVPWd, left ventricular posterior wall diameter; IVSd, interventricular septum diameter. 
(NM_000256.3), cardiac troponin I (TNNI3) (NM_000363.4), cardiac troponin $\mathrm{T}$ (TNNT2) (NM_001001430.1), myosin ventricular regulatory light chain 2 (MYL2) (NM_000432.3) and myosin ventricular essential light chain 1 (MYL3) (NM_000258.2) has been described previously. ${ }^{15} \mathrm{HCM}$ patients with mutations classified as variants of unknown significance were defined as genotype negative and family members of these were not included.

\section{Echocardiography}

Two-dimensional echocardiographic studies were performed on Vivid 7 or Vivid E9 machines (GE Healthcare, Horten, Norway). Data were analysed with EchoPAC version 112 (GE Healthcare). LV wall thickness was measured from parasternal short-axis view, and MWT was defined as the most hypertrophic segment in all LV segments from the base to the apex of the LV. ${ }^{1}$ In the HCM LVH+patients, we used the Maron classification (I-IV) to describe the phenotype. ${ }^{16}{ }^{17}$ According to the classification, type I was defined as anteroseptal hypertrophy, type II affecting the whole septum, type III the septum andat least part of the LV-free wall and type IV affecting other location as in the apex. The interventricular septal diameter, $L V$ posterior wall diameter, $L V$ end-diastolic diameter (LVEDD), LV end-systolic diameter (LVESD), left atrial diameter and LV mass (Cube formula) were determined by M-mode or 2-D imaging. ${ }^{18}$ Peak left ventricular outlet tract (LVOT) gradients were measured only at rest, and LVOT obstruction was defined as pressure gradient $\geq 30 \mathrm{~mm} \mathrm{Hg} .{ }^{1} \mathrm{LV}$ end-diastolic volume (EDV) and end-systolic volume (ESV) were measured and EF was calculated by Simpson's formula. ${ }^{18} \mathrm{LV}$ volumes were indexed by body surface area. Diastolic function was evaluated by transmitral pulsed Doppler and tissue Doppler. Early diastolic mitral annular velocity ( $\left.\mathrm{e}^{\prime}\right)$ by tissue Doppler in apical four-chamber view was calculated as the average of septal and lateral samplings. Atrial area was measured at end-systole and averaged from apical four-chamber and apical two-chamber views. ${ }^{18}$

Longitudinal strain by speckle tracking echocardiography was obtained from three apical views at frame rates $>50 / \mathrm{s}$. The endocardial border was traced in each view and speckles were tracked frame by frame during the cardiac cycle. Segments that failed to track were manually adjusted, and segments that subsequently failed to track were excluded. Region of interest was adjusted to fit the myocardial thickness. LV GLS was defined as the average of peak longitudinal strain from the $16 \mathrm{LV}$ segments model. ${ }^{19}$

\section{Statistical analyses}

Continuous data were presented as mean \pm SD. Comparisons of means between groups were performed by unpaired Student's t-test or analysis of variance F-test with Bonferroni post hoc correction when more than two groups were compared. Proportions were compared by $\chi^{2}$ test or Fisher's exact test (SPSS version 21). Indexed $\mathrm{LV}$ volumes were adjusted for gender and age by multivariate logistic regression. Correlations between EF, GLS, MWT, End-Systolic Volume Index (ESVI), $\mathrm{e}^{\prime}$ and cardiac volumes were assessed by linear regression analysis. Interobserver and intraobserver variabilities were expressed by intraclass correlation coefficients. Two-sided $p$ values $\leq 0.05$ were considered statistically significant.

Table 2 LV volumes, EF and diastolic parameters evaluated by echocardiography in 80 healthy individuals, 100 HCM LVHpatients and $180 \mathrm{HCM} \mathrm{LVH}+$ patients

\begin{tabular}{|c|c|c|c|c|}
\hline & $\begin{array}{l}\text { Healthy individuals } \\
(n=80)\end{array}$ & $\begin{array}{l}\text { HCM LVH- patients } \\
(n=100)\end{array}$ & $\begin{array}{l}\text { HCM LVH+ patients } \\
(n=180)\end{array}$ & p Value \\
\hline EDV/BSA $\left(\mathrm{mL} / \mathrm{m}^{2}\right)$ & $57 \pm 14$ & $49 \pm 13^{*}$ & $41 \pm 14^{* \dagger}$ & $<0.001$ \\
\hline $\mathrm{ESV} / \mathrm{BSA}\left(\mathrm{mL} / \mathrm{m}^{2}\right)$ & $23 \pm 9$ & $19 \pm 6^{*}$ & $16 \pm 7^{* \dagger}$ & $<0.001$ \\
\hline EF $(\%)$ & $61 \pm 6$ & $60 \pm 5$ & $61 \pm 7$ & 0.43 \\
\hline GLS (\%) & $-22.3 \pm 3.7$ & $-21.3 \pm 2.4$ & $-16.4 \pm 3.7^{\star \star \dagger}$ & $<0.001$ \\
\hline$E(m / s)$ & $0.7 \pm 0.2$ & $0.8 \pm 0.2$ & $0.8 \pm 0.8$ & 0.24 \\
\hline E/A ratio & $1.4 \pm 0.5$ & $1.6 \pm 0.6$ & $1.3 \pm 0.7^{\dagger}$ & 0.04 \\
\hline Deceleration time (ms) & $195 \pm 48$ & $175 \pm 30$ & $217 \pm 74^{*} \dagger$ & $<0.001$ \\
\hline $\mathrm{E} / \mathrm{e}^{\prime}$ & $6.4 \pm 2.1$ & $7.0 \pm 2.2$ & $14.9 \pm 8.3^{* \dagger}$ & $<0.001$ \\
\hline$e^{\prime}(m / s)$ & $0.12 \pm 0.03$ & $0.06 \pm 0.03^{*}$ & $0.11 \pm 0.04^{* \dagger}$ & $<0.001$ \\
\hline LA area $\left(\mathrm{cm}^{2}\right)$ & $17.0 \pm 4.1$ & $16.3 \pm 4.5$ & $24.9 \pm 11.3^{* \dagger}$ & $<0.001$ \\
\hline
\end{tabular}

The values are represented as mean $\pm \mathrm{SD}$.

$p$ values are based on Student's t-test.

${ }^{*} \mathrm{p}<0.05$ compared with healthy individuals.

$+\mathrm{p}<0.05$ compared with HCM LVH- patients.

A, atrial transmitral filling velocity; BSA, body surface area; $e^{\prime}$, early diastolic myocardial velocity; E, early transmitral flow velocity; EDV, enddiastolic volume; EF, ejection fraction; ESV, end-systolic volume; GLS, global longitudinal strain; HCM, hypertrophic cardiomyopathy; HCM LVH-, HCM patients without increased wall thickness; HCM LVH+, HCM patients with increased wall thickness; LA, left atrium; LV, left ventricle; LVH, left ventricular hypertrophy. 


\section{RESULTS}

\section{Patient characteristics and genetic analyses}

A total of $180 \mathrm{HCM} \mathrm{LVH}+$ patients, $100 \mathrm{HCM}$ LVH- patients and 80 healthy individuals were included in the study (table 1).

The HCM LVH+ patients were more frequently male compared with the HCM LVH- patients $(\mathrm{p}<0.01)$. The HCM LVH- patients were younger than the HCM LVH+ patients and the healthy individuals $(\mathrm{p}<0.001)$ (table 1$)$. Body mass index was higher in the HCM LVH+ patients than the HCM LVH- patients and the healthy individuals $(\mathrm{p}<0.001)$. HCM-related pathogenic mutations were confirmed in $90(50 \%) \mathrm{HCM} \mathrm{LVH}+$ patients $(59(66 \%)$ MYBP3, 25 (28\%) MYH7, 4 (4\%) TNNI3 and 2 (2\%) TNNT2) and in all $100 \mathrm{HCM}$ LVH- patients as defined in our inclusion criteria (58 (58\%) MYBP3, 29 (29\%) MYH7, $3(3 \%)$ TNNI3 and $4(4 \%)$ MYL3 and $6(6 \%)$ MYL2).

\section{General echocardiographical findings}

As expected, MWT was thicker and the LVOT gradient was higher in the HCM LVH+ patients compared with HCM LVH- patients and healthy individuals (all $\mathrm{p}<0.001$ ), but with no differences between the HCM LVH- patients and healthy individuals $(\mathrm{p}=1.0)$ (table 1$)$. In the HCM $\mathrm{LVH}+$ patients, $18.9 \%(\mathrm{n}=34)$ presented with a type $\mathrm{I}$, $40.0 \%(\mathrm{n}=72)$ with a type II, $37.2 \%(\mathrm{n}=67)$ type III and $3.9 \%(n=7)$ type IV hypertrophy pattern according to the Maron classification of HCM phenotype.

The HCM LVH+ patients had smaller LV cavities measured by LVEDD and LVESD compared with HCM LVH- patients $(\mathrm{p}=0.02$ and $\mathrm{p}<0.001)$ and

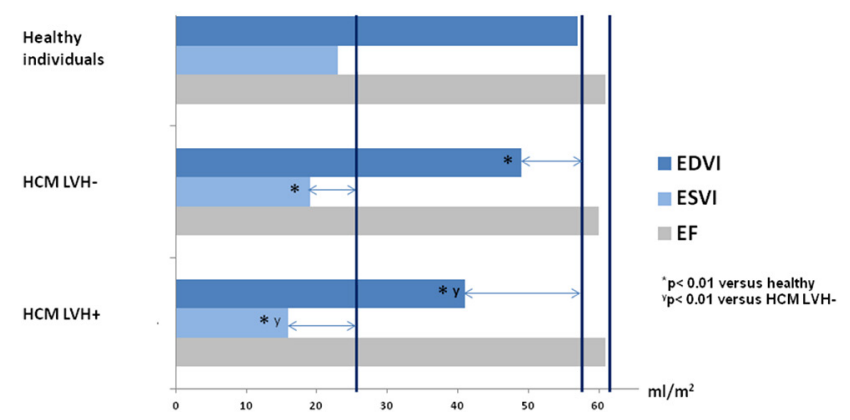

Figure 1 Bar chart of cardiac volumes and $\mathrm{EF}$ in the healthy individuals, HCM LVH- patients and HCM LVH+ patients. Blue bars show the indexed diastolic volumes $\left(E D V I\left(\mathrm{~mL} / \mathrm{m}^{2}\right)\right)$, light blue bars show the indexed systolic volumes (ESVI $\left.\left(\mathrm{mL} / \mathrm{m}^{2}\right)\right)$ and grey bars show EF (\%). The dark blue vertical lines are reference for average EDVI, ESVI and EF in healthy individuals. The horizontal arrows indicate the difference in average indexed diastolic and systolic volumes and EF between HCM LVH- and HCM LVH+ patients compared with healthy individuals. ${ }^{*} p<0.01$ versus healthy individuals, $\uparrow p<0.01$ versus HCM LVH-. EDVI, End-Diastolic Volume Index; EF, ejection fraction; HCM, hypertrophic cardiomyopathy; ESVI, End-Systolic Volume Index; HCM, hypertrophic cardiomyopathy; HCM LVH-, genotype-positive patients without increased wall thickness; HCM LVH+, patients with increased wall thickness; LVH, left ventricular hypertrophy. healthy individuals (both $\mathrm{p}<0.001$ ). HCM LVH- patients had smaller LVEDD and LVESD than healthy individuals $(\mathrm{p}=0.05$ and $\mathrm{p}=0.03$ ) (table 1 ). Indexed LV mass was significantly higher in the HCM LVH+ patients compared with the HCM LVH- patients and healthy individuals (both $p<0.001$ ), but with no differences between the HCM LVH- patients and healthy individuals $(p=1.0)$.

$\mathrm{HCM} \mathrm{LVH}+$ patients had increased $\mathrm{E}$ deceleration time, $\mathrm{E} / \mathrm{e}^{\prime}$ and atrial area compared with HCM LVH- patients and healthy individuals (all $\mathrm{p}<0.001$ ), whereas there were no differences between HCM LVH- patients and healthy individuals. HCM LVH- patients had reduced $\mathrm{e}^{\prime}$ compared with the healthy individuals $(\mathrm{p}=0.01)$ (table 2$)$.

\section{LV volumes and systolic function}

End-Diastolic Volume Index (EDVI) was significantlysmaller in HCM LVH+ patients than in HCM LVH- patients and healthy individuals (both $\mathrm{p}<0.001$ ) (table 2). Small EDVI was more likely in HCM LVH+ patients also when adjusting for gender and age, compared with HCM LVHpatients (OR 0.97 (95\% CI 0.95 to 0.99 ), $\mathrm{p}<0.01$ ) and compared with healthy individuals (OR 0.92 (95\% CI 0.89 to 0.94$), \mathrm{p}<0.001)$. ESVI was likewise significantly smaller in the HCM LVH+ patients compared with HCM LVH- patients and healthy individuals (both $\mathrm{p}<0.001$ ) (table 2). ESVI adjusted for gender and age was smaller in the HCM LVH+ patients compared with healthy individuals (OR 0.88 (95\% CI 0.84 to 0.92 ), $\mathrm{p}<0.001$ ), but not compared with HCM LVH- patients (OR 0.96 (95\% CI 0.91 to 1.00 ), $\mathrm{p}=0.09$ ). HCM LVH- patients had significantly lower EDVI and ESVI compared with healthy individuals (both $\mathrm{p}<0.001$ ) (table 2 ), also when adjusting for gender and age (OR 0.92 (95\% CI 0.89 to 0.94$)$ and (OR 0.88 (95\% CI 0.84 to 0.92 ), both $\mathrm{p}<0.001$ ) (figure 1 ).

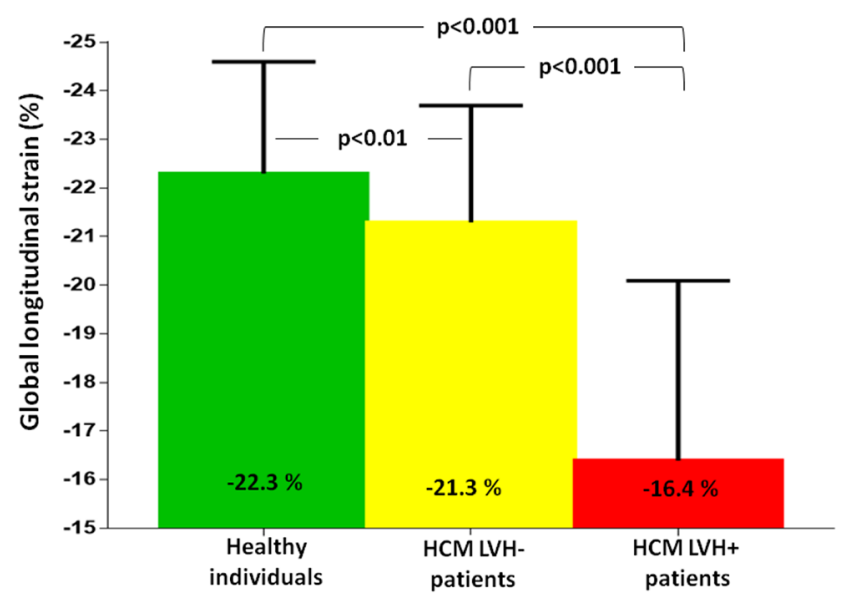

Figure 2 Bar chart of systolic function in healthy individuals, HCM LVH- patients and HCM LVH+ patients. GLS was significantly worse in the HCM LVH+ patients compared with HCM LVH- patients and healthy individuals (both $\mathrm{p}<0.001$ ). In addition, GLS was worse in the HCM LVH- compared with healthy individuals $(p=0.005)$. GLS, global longitudinal strain; HCM, hypertrophic cardiomyopathy; HCM LVH-, genotype-positive patients without increased wall thickness; HCM LVH+, patients with increased wall thickness; LVH, left ventricular hypertrophy. 

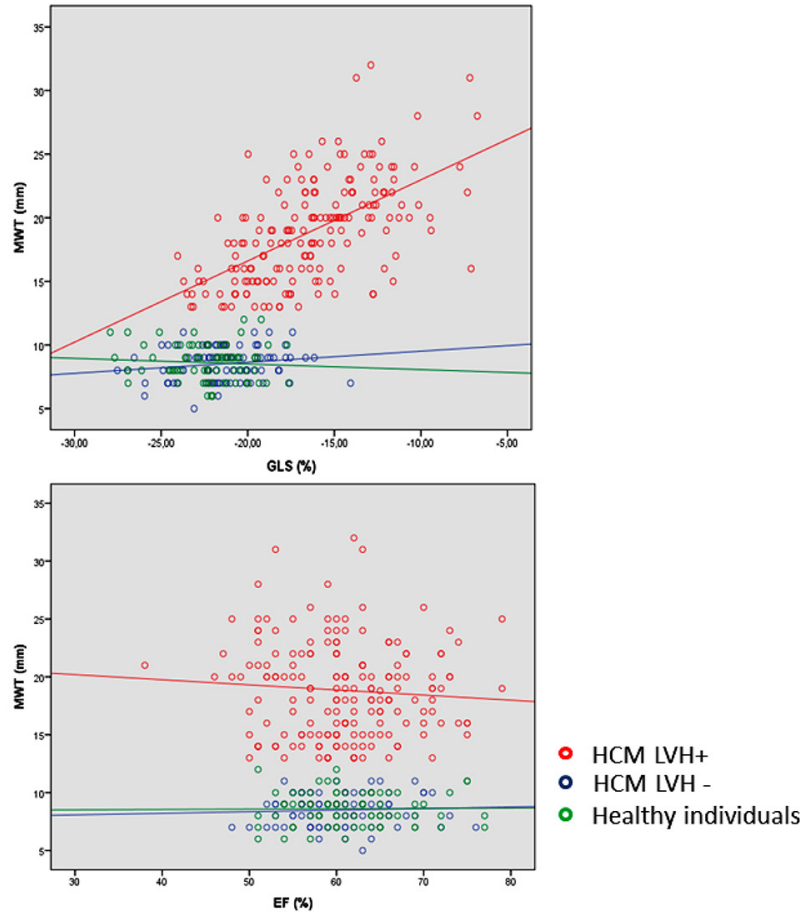

Figure 3 Scatter plot of relationship between MWT, GLS and EF upper panel shows that GLS was closely related to MWT with worse LV function by GLS correlating to increased wall thickness $(R=0.58, p<0.001)$ in the HCM $\mathrm{LVH}+$ patients (red dots), but with no correlation between GLS and MWT $(R=0.16, p=0.13)$ in the HCM LVH- (blue dots) and the healthy individuals $(R=0.07, p=0.53)$ (green dots). Lower panel shows that there was no significant correlation between EF and MWT in the HCM LVH+ patients $(\mathrm{R}=0.018, \mathrm{p}=0.30)$ or in the HCM LVH- patients and the healthy individuals $(R=0.05, p=0.60$ and $R=0.01, p=0.91)$. EF, ejection fraction; GLS, global longitudinal strain; HCM $\mathrm{LVH}+$, hypertrophic cardiomyopathy patients with hypertrophy; HCM LVH-, genotype-positive patients without hypertrophy; MWT, maximal wall thickness.

EF did not differ between the HCM LVH+, HCM LVH- and healthy individuals $(\mathrm{p}=0.43)$ (table 2), but systolic function evaluated by GLS was worse in HCM LVH+ patients compared with HCM LVH- patients $(-16.4 \% \pm 3.7 \%$ vs $-21.3 \% \pm 2.4 \%, \mathrm{p}<0.001)$ and healthy individuals $(-22.3 \% \pm 3.7 \%, \mathrm{p}<0.001)$, indicating reduced longitudinal LV systolic function despite normal EF (figure 2). The mutation-negative HCM LVH+ patients had significantly worse GLS than the mutation-positive patients $(-15.2 \% \pm 3.4 \%$ vs $-17.6 \% \pm 3.4 \%, \mathrm{p}<0.001)$, but there were no differences in GLS related to the pathogenic mutations. The GLS was significantly worse with increased distribution of hypertrophy $(-18.9 \% \pm 2.8 \%$ in type I, $-16.5 \% \pm 3.2 \%$ in type II, $-14.7 \% \pm 3.7 \%$ in type III and $-19.3 \% \pm 2.8 \%$ in type IV) with significantly worse GLS in type III compared with the other hypertrophy pattern $(-14.7 \% \pm 3.7 \%$ vs $-17.3 \pm 3.3 \%, \mathrm{p}<0.001)$.

GLS was worse in the HCM LVH- patients compared with healthy individuals in pairwise comparison $(p=0.001)$ demonstrating subtle changes in systolic function also in the HCM LVH- patients (table 2) (figure 2).
A lower ESVI was closely related to EF in HCM LVH+ and HCM LVH- patients $(\mathrm{R}=0.45, \mathrm{p}<0.001$ and $\mathrm{R}=0.43$, $\mathrm{p}<0.001)$ as expected, but there was no relationship with GLS ( $\mathrm{R}=0.02, \mathrm{p}=0.77$ and $\mathrm{R}=0.11, \mathrm{p}=0.31)$. Increased MWT correlated significantly with worse GLS $(\mathrm{R}=0.58$, $\mathrm{p}<0.001)$, but not with $\mathrm{EF}(\mathrm{R}=0.018, \mathrm{p}=0.30)$ in the HCM $\mathrm{LVH}+$ patients. There were no correlation between MWT and GLS or EF in the HCM LVH- patients $(\mathrm{R}=0.16$, $\mathrm{p}=0.13$ and $\mathrm{R}=0.05, \mathrm{p}=0.60$ ) and in the healthy individuals $(\mathrm{R}=0.07, \mathrm{p}=0.53$ and $\mathrm{R}=0.01, \mathrm{p}=0.91$ ) (figure 3$)$.

In the HCM LVH+ population, lower EDVI and ESVI were significantly related to lower $\mathrm{e}^{\prime}(\mathrm{R}=0.24, \mathrm{p}=0.05$ and $\mathrm{R}=0.21, \mathrm{p}=0.01$ ) and lower $\mathrm{e}^{\prime}$ was significantly correlated to worse GLS $(\mathrm{R}=0.47, \mathrm{p}<0.001)$. There was a weak correlation between cardiac volumes and lower $\mathrm{e}^{\prime}$ in the HCM LVH- patients $(\mathrm{R}=0.27, \mathrm{p}=0.02$ for EDVI and $\mathrm{R}=0.32$, $\mathrm{p}=0.04$ for ESVI, respectively), but with no correlation between $\mathrm{e}^{\prime}$ and GLS $(\mathrm{R}=0.03, \mathrm{p}=0.79)$.

\section{Feasibility and variability analyses}

LV volume measurements could be performed in all patients and healthy individuals with intraobserver and interobserver intraclass correlation coefficient of 0.97 (95\% CI 0.87 to 0.99 ) and 0.92 (95\% CI 0.69 to 0.98 ) for EDV and 0.97 (95\% CI 0.88 to 0.99 ) and 0.93 (95\% CI 0.72 to 0.98 ) for ESV, respectively.

Strain analysis could be performed in 176 (98\%) HCM $\mathrm{LVH}+$ patients, $89(89 \%)$ HCM LVH- patients and 78 $(98 \%)$ healthy individuals. Ninety per cent of the LV segments were included in strain analyses in HCM LVH+ and HCM LVH- patients and 94\% of LV segments in healthy individuals. Intraobserver and interobserver intraclass correlation coefficients were 0.90 (95\% CI 0.61 to 0.98 ) and 0.91 (95\% CI 0.72 to 0.97 ), respectively, for strain measurements.

\section{DISCUSSION}

Our study demonstrated a close relationship between volume changes and $\mathrm{EF}$ in patients with $\mathrm{HCM}$, whereas GLS was related to MWT. Volumetric changes and reduced diastolic and systolic function were present also in HCM LVH- patients with smaller cardiac volumes, reduced $\mathrm{e}^{\prime}$ and worse GLS compared with healthy individuals despite normal cardiac wall thickness, indicating a continuum of volumetric and systolic changes from the healthy individuals to HCM LVH- and HCM LVH+ patients.

The chain of mechanisms in HCM disease is complex and is influenced by several molecular changes and haemodynamic alterations. Our results imply that the initial changes in HCM are characterised by a gradually less compliant and stiffer LV which contributes to the decreased systolic long axis function and diastolic dysfunction before the occurrence of visible morphological changes. As HCM disease progresses, the continued increase in wall thickness and reduced cavity will further aggravate LV long axis dysfunction and prevent normal 
LV filling, whereas the reduced cavity size will lead to normal values of $\mathrm{EF}$.

\section{Volume and myocardial compliance}

This study demonstrated reduced LV volumes in HCM $\mathrm{LVH}+$ patients compared with HCM LVH- patients and healthy individuals in line with previous studies. ${ }^{20}$ The reduced LV volumes may have several explanations. Increased MWT will lead to decreased size of the heart cavity, both in systole and in diastole. However, the decreased volume cannot be attributed to the increase in MWT alone. As expected, diastolic function was reduced in our HCM LVH+ patients compared with HCM LVHpatients and healthy individuals. Diastolic dysfunction was significantly related to cardiac volumes with a significant correlation between lower $\mathrm{e}^{\prime}$ and lower cardiac volumes confirming the relationship between decreased LV filling and reductions in cardiac volumes in $\mathrm{HCM} \mathrm{LVH}+{ }^{12}$

Our results showed reduced LV volumes also in the HCM LVH- patients with normal wall thickness. Captur et al showed reduced ESV in HCM LVH- patients by cardiovascular magnetic resonance (CMR) supporting our findings. ${ }^{21}$ The mechanisms for reduced volumes in HCM LVH- are less clear. We know that reduced ventricular compliance will move the Frank-Starling pressure-volumecurve leftward and the end-diastolic pressure and preload become higher despite smaller EDV. ${ }^{22}$ HCM LVH- patient had reduced $\mathrm{e}^{\prime}$ compared with healthy individuals, and we speculate if higher LV end-diastolic pressures and decreased LV filling may be a possible explanation for the reduced diastolic and systolic volumes even in this early stage of disease. ${ }^{23}$ Despite changes in LV volume and $\mathrm{e}^{\prime}$, there were no significant differences in diastolic parameters as deceleration time, $\mathrm{E} / \mathrm{e}^{\prime}$ and left atrium area between healthy individuals and HCM LVH- patients. However, the diastolic variances can be underestimated because of the significant age difference with younger HCM LVH- patients. This is a limitation in our study.

\section{Myocardial function and wall thickness}

Not surprisingly, EF was similar in both HCM groups compared with healthy individuals despite severely reduced systolic function by GLS in our HCM LVH+ patients. The preserved EF in the HCM LVH+ could be explained by the smaller volumes in HCM, which directly influence on the EF equation. Afterload is a major determinant of systolic performance, and according to Laplace law, directly related to LV systolic pressure and radius and inversely related to wall thickness as typically found in hypertension and in aortic stenosis maintaining normal EF. ${ }^{24}$ However, in HCM LVH+ patients, the hypertrophy is the pathophysiological cause of the disease and the reduction in afterload due to ventricular wall changes cannot be directly attributed to changes in systolic function found in our patients. ${ }^{25}$ We believe that reduced systolic function by GLS in the HCM LVH+ patients may be explained by reduced LV compliance and myocardial fibrosis. It has previously been shown that diastolic and longitudinal systolic LV function by GLS is more depressed in $\mathrm{HCM} \mathrm{LVH}+$ patients with fibrosis by $\mathrm{CMR}$. 72627

The distribution of hypertrophy according to Maron classification in our HCM LVH+ population with worse GLS in type III phenotypeisin line with previous publication by Reant et al. ${ }^{17}$ Worse LV function by GLS was closely related to increased MWT, whereas EF was not related to MWT in our study. Increased wall thickness is a good indicator of the staging of disease, and our results suggest that GLS is a sensitive parameter for detecting systolic impairment related to increased wall thickness, in contrast to EF. GLS should therefore be evaluated at every visit in patients with HCM .

GLS was reduced in the HCM LVH- compared with the healthy individuals. Cardim et al have showed that myocardial longitudinal velocities and deformation parameters may be abnormal before the development of increased wall thickness and to be a hallmark in HCM LVH- patients. ${ }^{11}$ Our results with the subtle changes in cardiac volumes, reduced $\mathrm{e}^{\prime}$ and worse GLS compared with healthy individuals confirm the occurrence of myocardial dysfunction in HCM LVH-. The hypertrophy can therefore be a compensatory mechanism for the mutation induced abnormalities. ${ }^{28} 29$

We speculate if interstitial fibrosis can contribute to deteriorated GLS, diastolic dysfunction and smaller cardiac volumes in our HCM LVH- patients compared with healthy individuals. Our study could not address the impact of myocardial fibrosis assessed by CMR on myocardial function, however, studies on precontrast and postcontrast myocardial relaxation time $\left(\mathrm{T}_{1}\right)$ mapping by CMR have demonstrated increased interstitial fibrosis independent of LV hypertrophy. Interstitial fibrosis may therefore be an early consequence of sarcomere mutations, and not only a downstream response to LV hypertrophy. ${ }^{30}$ Although cardiac volumes were significantly smaller and GLS worse in HCM LVH- compared with healthy individuals, measurements were within normal ranges, which makes GLS difficult to use as a parameter to distinguish them from the healthy individuals.

\section{Clinical implications}

Future studies should investigate if HCM LVH- patients with the smallest cardiac volumes and with worse GLS are more likely to develop a HCM LVH+ phenotype with increased wall thickness later in life. We are in need of longitudinal follow-up data to confirm our findings, but we suggest that HCM LVH- with volumetric and subtle diastolic and systolic dysfunction should be followed as closely as the HCM LVH+ patients according to the guidelines. ${ }^{1}$

\section{Study limitations}

Volume and strain measurements are dependent on good image quality as all echocardiographic measurements. 
The use of 2-D echocardiography to calculate $\mathrm{LV}$ volumes in HCM patients with increased MWT and asymmetry of the LV chamber is challenging and is a limitation of our findings. In addition, it can be challenging to fit the region of interest for strain analyses correctly in HCM patients due to variable wall thickness. In our population, gender and age were unequally distributed between groups, with a male to female ratio of 1.4:1 and higher age in the HCM LVH+ population. This is in line with previous reports, showing higher penetrance of HCM phenotype in males with increasing age. ${ }^{31}$

\section{CONCLUSION}

The preserved EF was related to smaller cardiac volumes in HCM LVH+ patients. Worse GLS by strain echocardiography correlated with increased MWT and is therefore a good parameter staging the HCM disease. HCM LVHpatients had subtle changes with reduced cardiac volumes and systolic function compared with healthy individuals, indicating a continuum of volumetric and systolic changes before wall thickness increases above normal.

Acknowledgements We thank all the individuals who participated in this study. Contributors All authors have read and approved the manuscript.

Funding This work was supported by the Center for Cardiological Innovation, funded by the Research Council of Norway.

Competing interests None declared.

Ethics approval The study complied with the Declaration of Helsinki and was approved by the Regional Committees for Medical Research Ethics.

Provenance and peer review Not commissioned; externally peer reviewed.

Data sharing statement № available unpublished data.

Open Access This is an Open Access article distributed in accordance with the Creative Commons Attribution Non Commercial (CC BY-NC 4.0) license, which permits others to distribute, remix, adapt, build upon this work non-commercially, and license their derivative works on different terms, provided the original work is properly cited and the use is non-commercial. See: http://creativecommons.org/ licenses/by-nc/4.0/

(c) Article author(s) (or their employer(s) unless otherwise stated in the text of the article) 2017. All rights reserved. No commercial use is permitted unless otherwise expressly granted.

\section{REFERENCES}

1. Elliott PM, Anastasakis A, Borger MA, et al. Authors/Task Force members. 2014 ESC Guidelines on diagnosis and management of hypertrophic cardiomyopathy: the Task Force for the Diagnosis and Management of Hypertrophic Cardiomyopathy of the European Society of Cardiology (ESC). Eur Heart J 2014;35:2733-79.

2. Afonso LC, Bernal J, Bax JJ, et al. Echocardiography in hypertrophic cardiomyopathy: the role of conventional and emerging technologies. JACC Cardiovasc Imaging 2008;1:787-800.

3. Cardim N, Galderisi M, Edvardsen T, et al. Role of multimodality cardiac imaging in the management of patients with hypertrophic cardiomyopathy: an expert consensus of the European Association of Cardiovascular Imaging endorsed by the Saudi Heart Association. Eur Heart J Cardiovasc Imaging 2015;16:280.laboratories, and age matched with HCM LVH+ patients.All participants gave written informed consent. Thestudy complied with the Declaration of Helsinki andwas approved by the Regional Committees for MedicalResearch Ethicslaboratories, and age matched with HCM $\mathrm{LVH}+$ patients.All participants gave written informed consent. Thestudy complied with the Declaration of Helsinki andwas approved by the Regional Committees for MedicalResearch Ethics

4. Edvardsen T, Haugaa KH. Imaging assessment of ventricular mechanics. Heart 2011;97:1349-56.
5. Almaas VM, Haugaa KH, Strøm EH, et al. Increased amount of interstitial fibrosis predicts ventricular arrhythmias, and is associated with reduced myocardial septal function in patients with obstructive hypertrophic cardiomyopathy. Europace 2013;15:1319-27.

6. De S, Borowski AG, Wang $\mathrm{H}$, et al. Subclinical echocardiographic abnormalities in phenotype-negative carriers of myosin-binding protein $\mathrm{C} 3$ gene mutation for hypertrophic cardiomyopathy. Am Heart J 2011;162:262-7. e263.

7. Almaas VM, Haugaa KH, Strøm EH, et al. Noninvasive assessment of myocardial fibrosis in patients with obstructive hypertrophic cardiomyopathy. Heart 2014;100:631-8.

8. Serri K, Reant P, Lafitte M, et al. Global and regional myocardial function quantification by two-dimensional strain: application in hypertrophic cardiomyopathy. J Am Coll Cardiol 2006;47:1175-81.

9. Ozawa K, Funabashi N, Takaoka H, et al. Characteristic myocardial strain identified in hypertrophic cardiomyopathy subjects with preserved left ventricular ejection fraction using a novel multilayer transthoracic echocardiography technique. Int $J$ Cardiol 2015;184:237-43.

10. Reant P, Mirabel M, Lloyd G, et al. Global longitudinal strain is associated with heart failure outcomes in hypertrophic cardiomyopathy. Heart 2016;102:741-7.

11. Cardim N, Perrot A, Ferreira T, et al. Usefulness of Doppler myocardial imaging for identification of mutation carriers of familial hypertrophic cardiomyopathy. Am J Cardiol 2002;90:128-32.

12. Nagueh SF, Bachinski LL, Meyer D, et al. Tissue Doppler imaging consistently detects myocardial abnormalities in patients with hypertrophic cardiomyopathy and provides a novel means for an early diagnosis before and independently of hypertrophy. Circulation 2001;104:128-30.

13. Peyrou J, Réant $P$, Reynaud A, et al. Morphological and functional abnormalities pattern in hypertrophy-free HCM mutation carriers detected with echocardiography. Int J Cardiovasc Imaging 2016;32:1379-89.

14. Olivotto I, Maron MS, Autore C, et al. Assessment and significance of left ventricular mass by cardiovascular magnetic resonance in hypertrophic cardiomyopathy. J Am Coll Cardiol 2008;52:559-66.

15. Berge KE, Leren TP. Genetics of hypertrophic cardiomyopathy in Norway. Clin Genet 2014;86:355-60.

16. Maron BJ, Gottdiener JS, Epstein SE. Patterns and significance of distribution of left ventricular hypertrophy in hypertrophic cardiomyopathy. A wide angle, two dimensional echocardiographic study of 125 patients. Am J Cardiol 1981;48:418-28.

17. Reant $P$, Donal $E$, Schnell $F$, et al. Clinical and imaging description of the Maron subtypes of hypertrophic cardiomyopathy. Int $J$ Cardiovasc Imaging 2015;31:47-55.

18. Lang RM, Badano LP, Mor-Avi V, et al. Recommendations for cardiac chamber quantification by echocardiography in adults: an update from the American Society of Echocardiography and the European Association of Cardiovascular Imaging. Eur Heart J Cardiovasc Imaging 2015;16:233-71.

19. Cerqueira MD, Weissman NJ, Dilsizian V, et al. Standardized myocardial segmentation and nomenclature for tomographic imaging of the heart. A statement for healthcare professionals from the cardiac imaging committee of the council on clinical cardiology of the American Heart Association. Circulation 2002;105:539-42.

20. Roşca M, Călin A, Beladan CC, et al. Right ventricular remodeling, its correlates, and its clinical impact in hypertrophic cardiomyopathy. $J$ Am Soc Echocardiogr 2015;28:1329-38.

21. Captur G, Lopes LR, Mohun TJ, et al. Prediction of sarcomere mutations in subclinical hypertrophic cardiomyopathy. Circ Cardiovasc Imaging 2014;7:863-71.

22. Gaasch WH, Levine HJ, Quinones MA, et al. Left ventricular compliance: mechanisms and clinical implications. Am J Cardiol 1976;38:645-53.

23. Pak PH, Maughan L, Baughman KL, et al. Marked discordance between dynamic and passive diastolic pressure-volume relations in idiopathic hypertrophic cardiomyopathy. Circulation 1996;94:52-60.

24. Lorell BH, Carabello BA. Left ventricular hypertrophy: pathogenesis, detection, and prognosis. Circulation 2000;102:470-9.

25. Maron BJ. Contemporary insights and strategies for risk stratification and prevention of sudden death in hypertrophic cardiomyopathy. Circulation 2010;121:445-56.

26. Urbano-Moral JA, Rowin EJ, Maron MS, et al. Investigation of global and regional myocardial mechanics with 3-dimensional speckle tracking echocardiography and relations to hypertrophy and fibrosis in hypertrophic cardiomyopathy. Circ Cardiovasc Imaging 2014;7:11-19.

27. Motoyasu M, Kurita T, Onishi K, et al. Correlation between late gadolinium enhancement and diastolic function in hypertrophic 
cardiomyopathy assessed by magnetic resonance imaging. Circ $J$ 2008;72:378-83

28. Ho CY, López B, Coelho-Filho OR, et al. Myocardial fibrosis as an early manifestation of hypertrophic cardiomyopathy. N Engl J Med 2010;363:552-63.

29. Germans T, Rüssel IK, Götte MJ, et al. How do hypertrophic cardiomyopathy mutations affect myocardial function in carriers with normal wall thickness? Assessment with cardiovascular magnetic resonance. J Cardiovasc Magn Reson 2010;12:13.
30. Ho CY, Abbasi SA, Neilan TG, et al. T1 measurements identify extracellular volume expansion in hypertrophic cardiomyopathy sarcomere mutation carriers with and without left ventricular hypertrophy. Circ Cardiovasc Imaging 2013;6:415-22.

31. Christiaans I, Birnie E, Bonsel GJ, et al. Manifest disease, risk factors for sudden cardiac death, and cardiac events in a large nationwide cohort of predictively tested hypertrophic cardiomyopathy mutation carriers: determining the best cardiological screening strategy. Eur Heart J 2011;32:1161-70. 